\title{
Essential role of paternal chromatin in the regulation of transcriptional activity during mouse preimplantation development
}

\author{
Hong-Thuy Bui ${ }^{1,2}$, Sayaka Wakayama ${ }^{2}$, Eiji Mizutani ${ }^{2}$, Keun-Kyu Park$^{1}$, Jin-Hoi Kim ${ }^{1}$, \\ Nguyen Van Thuan ${ }^{1,2}$ and Teruhiko Wakayama ${ }^{2}$ \\ ${ }^{1}$ Department of Animal Biotechnology, College of Animal Bioscience and Biotechnology/Animal Resources Research \\ Center, Konkuk University, 1 Hwayang-dong, Gwangjin-gu, Seoul 143-701, Republic of Korea and ${ }^{2}$ Riken Center for \\ Developmental Biology, 2-2-3 Minatojima-minamimachi, Kobe 650-0047, Japan
}

Correspondence should be addressed to H-T Bui at Department of Animal Biotechnology, College of Animal Bioscience and Biotechnology/Animal Resources Research Center, Konkuk University; Email: bthuy@konkuk.ac.kr

N Van Thuan at Department of Animal Biotechnology, College of Animal Bioscience and Biotechnology/Animal Resources Research Center, Konkuk University; Email: vanthuan@konkuk.ac.kr

\begin{abstract}
Several lines of evidence indicate that the formation of a transcriptionally repressive state during the two-cell stage in the preimplantation mouse embryo is superimposed on the activation of the embryonic genome. However, it is difficult to determine the profile of newly synthesized (nascent) RNA during this phase because large amounts of maternal RNA accumulate in maturing oocytes to support early development. Using 5-bromouridine-5' -triphosphate labeling of RNA, we have verified that nascent RNA synthesis was repressed between the two-cell and four-cell transition in normally fertilized but not in parthenogenetic embryos. Moreover, this repression was contributed by sperm (male) chromatin, which we confirmed by studying androgenetic embryos. The source of factors responsible for repressing nascent RNA production was investigated using different stages of sperm development. Fertilization with immature round spermatids resulted in a lower level of transcriptional activity than with ICSI at the two-cell stage, and this was consistent with further repression at the four-cell stage in the ICSI group. Finally, study on DNA replication and chromatin remodeling was performed using labeled histones $\mathrm{H} 3$ and $\mathrm{H} 4$ to differentiate between male and female pronuclei. The combination of male and female chromatin appeared to decrease nascent RNA production in the fertilized embryo. This study indicates that paternal chromatin is important in the regulation of transcriptional activity during mouse preimplantation development and that this capacity is acquired during spermiogenesis.

Reproduction (2011) $14167-77$
\end{abstract}

\section{Introduction}

Some studies have shown that a transcriptionally repressive state develops during the two-cell stage in mouse embryos (Majumder et al. 1993, Henery et al. 1995, Davis et al. 1996, Aoki et al. 1997). Ma et al. (2001) reported that of more than 200 amplicons analyzed, using an mRNA differential display, about $45 \%$ were repressed between the two-cell and four-cell stage. They suggested that genome activation is global at this stage and that a function of the transcriptionally repressive state is to dictate the appropriate profile of gene expression that is compatible with further development. However, by the two-cell stage, the embryo forms the need for an enhancer for the efficient expression of a plasmid-borne luciferase reporter gene driven by the $t k$ promoter (Martinez-Salas et al. 1989, Wiekowski et al. 1991, Henery et al. 1995). This requirement appears to relieve the transcriptional repression that develops during the two-cell stage (Majumder \& DePamphilis 1995, Nothias et al. 1995).

Actually, in early preimplantation embryos the total RNA profile is likely to be very different from that of newly synthesized (nascent) RNA, as there is already a large accumulation of maternally derived RNA in the oocyte cytoplasm dedicated to supporting early embryogenesis. In addition, these maternally derived RNAs are rapidly degraded after fertilization, so any changes in the amounts of some RNA species might merely reflect the degradation of maternally derived RNA, while any ongoing de novo synthesis would be masked. However, previous reports on such nascent RNA dealt only with the two-cell stage in mouse embryos (Aoki et al. 1997, Kageyama et al. 2004). There has been no report about changes during the later stages, especially during 
the transition from the two-cell to the four-cell stage, when the nuclear chromatin becomes assembled into pro-chromocenters representing epigenetic marks characteristic of silenced chromatin (Martin et al. 2006). Such chromatin remodeling during the one-cell and twocell embryo stages might provide an opportunity for transcription factors to gain access to their cis-cognate DNA-binding sequences (Ma et al. 2001). Histone acetylation is important for chromatin remodeling and transcription. It alters nucleosomal conformation, which can increase the accessibility of transcriptional regulatory proteins to chromatin templates (Lee et al. 1993, Vettese-Dadey et al. 1996).

After fertilization, the paternal chromatin is remodeled exhaustively, the sperm nucleus loses its unique protamine-rich chromatin conformation, and the DNA is repopulated with maternally derived nucleosomes. In some mammalian species, including the mouse and human, but not the sheep or rabbit, the zygotic paternal genome is demethylated widely soon after fertilization, whereas the maternal genome remains highly methylated (Mayer et al. 2000, Young \& Beaujean 2004). This asymmetric patterning of DNA methylation in the two parental genomes at the one-cell stage persists at least until the two-cell embryo (Barton et al. 2001) and is believed responsible for the functional differences between the parental genomes during development. Recently, our laboratory found that the male germ cell acquires the ability to maintain its undermethylated state in the paternal zygotic genome during spermiogenesis, independent of the acquisition of oocyte-activating ability. Our study clearly showed that the paternal zygotic genome derived from a round spermatid is highly remethylated before the first mitosis after demethylation (Kishigami et al. 2006). Round spermatids are haploid cells of the spermatogenic line. During spermiogenesis, they undergo a major biochemical and morphological restructuring of the germ cell in which most of the somatic histones are replaced by protamines to pack the DNA into the sperm cell nucleus (Martianov et al. 2002). Obviously, mature spermatozoa are more successful in the reprograming of paternal zygotic genomes than immature forms. However, their contribution to the regulation of transcriptional activity during mouse preimplantation development has not been elucidated. In addition, our recent report showed that the success of somatic nuclear reprograming and cloning efficiency using somatic cell nuclear transfer (SCNT) techniques is clearly linked to the competent de novo synthesis of nascent RNA in SCNT-generated mouse clone embryos (Van Thuan et al. 2009). Therefore, in this study, we have investigated the changes in transcriptional activity during mouse embryo preimplantation development. We also examined the differences between male and female chromatin remodeling, along with DNA synthesis, nascent RNA production, and histone modifications. We found that a transcriptionally repressive stage was induced by the paternal chromatin during embryonic gene activation and that ICSI could regulate zygotic transcriptional activity successfully during preimplantation development.

\section{Results \\ Nascent RNA production is repressed in four-cell ICSI embryos, but is maintained in four-cell parthenogenetic embryos}

It has been suggested that chromatin-mediated repression is established during the formation of a two-cell mouse embryo and that this repression increases during the transition from the two-cell to the four-cell stage (Wiekowski et al. 1997). However, it is unclear whether the synthesis of nascent RNA is repressed during this phase. Therefore, we examined the levels of nascent RNA produced in ICSI-generated and parthenogenetic preimplantation embryos.

A high level of nascent RNA was detected in 95\% of the nuclei of parthenogenetic two-cell embryos (Figs 1A and 2). This high level was maintained in $90 \%$ of the nuclei of four-cell embryos (Figs 2 and 3A). For ICSIgenerated embryos, a high level of nascent RNA was also detected in $78 \%$ of the nuclei of two-cell embryos (Figs 1D and 2). It decreased quickly in the late two-cell embryos so that only $8 \%$ of the four-cell embryo stage nuclei showed high levels of nascent RNA (Figs 2 and $3 \mathrm{D})$. Thus, there were significant differences in transcriptional activity between ICSI-generated and parthenogenetic embryos at the four-cell stage.

There were similar levels of nascent RNA production in the two-cell and four-cell stages in ICSI-generated and in vivo fertilization-generated embryos (data not shown). This confirms that there was no influence of micromanipulation on the level of nascent RNA synthesis. In all of the experimental groups, negative control embryos were treated with $\alpha$-amanitin and did not show 5-bromouridine-5'-triphosphate (BrUTP) signals that would indicate RNA incorporation of this marker.

\section{Essential role of paternal chromatin in the repression of nascent RNA production}

As nascent RNA production was repressed during the two-cell to four-cell transition in ICSI-generated but not in parthenogenetic embryos, this led us to suspect that the paternal chromatin might cause transcriptional repression. We used experimental androgenesis to investigate this. A high level of newly synthesized RNA was detected in $36 \%$ of two-cell androgenetic embryo nuclei (Figs 1G and 2). This decreased quickly in most of the four-cell embryo nuclei (Figs 2 and 3G). There were similar levels of transcriptional repression at the four-cell stage in ICSI-generated and androgenetic embryos: most of these showed low transcriptional activity, whereas 


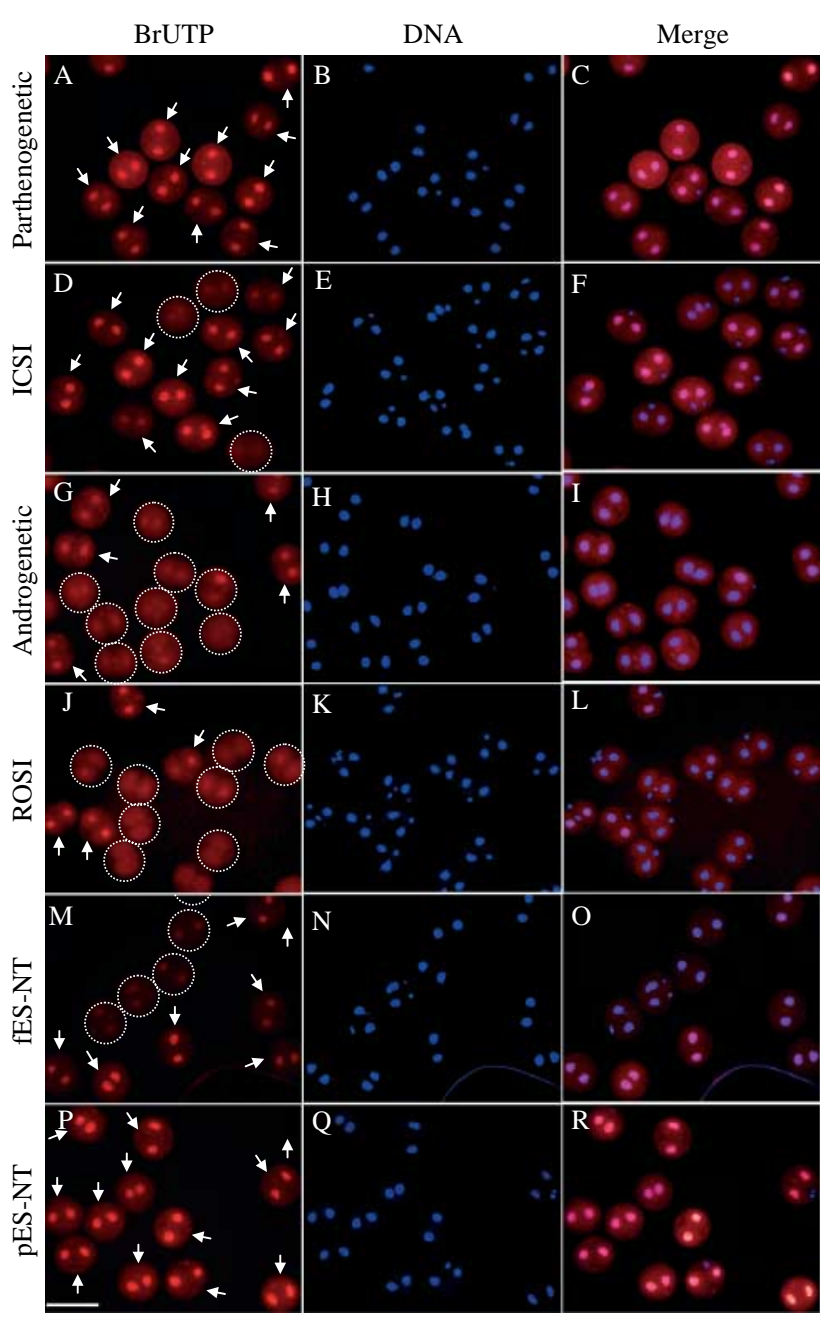

Figure 1 Nascent RNA expression at the two-cell stage in (A-C) parthenogenetic embryos, (D-F) ICSI-generated, (G-I) ICSI-generated androgenetic and (J-L) round spermatid injection (ROSI)-generated embryos, and in cloned embryos reconstructed by nuclear transfer (NT) from $(\mathrm{M}-\mathrm{O})$ fertilized embryonic stem ( $\mathrm{fES}$ ) cell and (P-R) parthenogenetic $(\mathrm{pES})$ cell nuclei. Embryos selected at $24 \mathrm{~h}$ were labeled with BrUTP and collected for the detection of BrU-labeled RNA transcripts using immunofluorescence microscopy. Nascent RNA is shown in red. The DNA was counterstained with 4,6-diamidino-2-phenylindole (DAPI; blue). The arrows indicate high levels of nascent RNA and circles indicate areas with low levels of nascent RNA. Bar $=100 \mu \mathrm{m}$.

most of the parthenogenetic embryos maintained a high level (Fig. 2). We further tested the idea that paternal factors might be involved in transcriptional repression by checking nascent RNA production in reconstructed cloned embryos by NT of mature oocytes with normally fertilized embryonic stem (fES) cells or parthenogenetic embryonic stem $(\mathrm{pES})$ cells, because fES cells contain both paternal and maternal genomes while pES cells contain only the maternal genome. If paternal factors influence transcriptional repression, then there should be differences in outcomes between these two kinds of embryos. When generating cloned embryos by NT with
ES cells in general, the majority arrest at the one-cell stage, which is different from those derived from the cumulus cell nuclei. This can be explained by the unique cell cycle of ES cells, which devote more than half of their entire cell cycle to the $S$ phase and have short $G_{1}$ and $\mathrm{G}_{2}$ phases (Stead et al. 2002). Although only a few embryos reached the two-cell stage (Supplementary Table 1, see section on supplementary data given at the end of this article), there were significant differences in nascent RNA production between the pES- and fESderived cloned embryos. A high level of nascent RNA was detected in $90 \%$ of two-cell embryo nuclei in the pES-NT group versus $64 \%$ of the fES-NT clones at this stage (Figs 1M, P, and 2; Supplementary Table 1). Interestingly, this high level was maintained in $86 \%$ of the four-cell embryo nuclei in the pES-NT clones while it decreased to $37 \%$ in the fES-NT group (Figs 2 and $3 \mathrm{M}$ and P). Taken together, these results suggest that malederived factors must be involved in suppressing nascent RNA production in the two-cell to four-cell transition in mouse embryos.

The similar levels of nascent RNA production in pathenogenetic and pES-NT in the two-cell and four-cell embryos reinforce that there was no influence of micromanipulation on the level of nascent RNA synthesis.

\section{Mature spermatozoa can regulate transcriptional activity successfully during preimplantation development}

We suspected that factors in the paternal chromatin must be involved in suppressing nascent RNA production in early mouse embryos. In the next experiment,

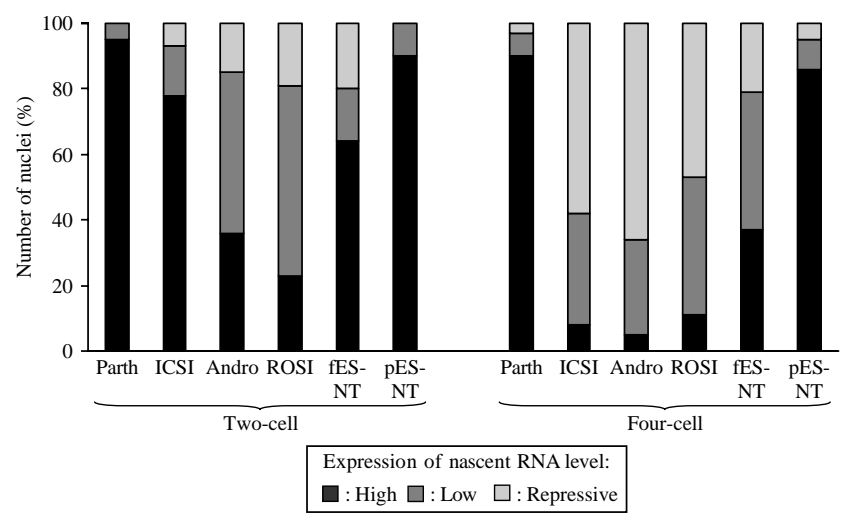

Figure 2 Nascent RNA expression in parthenogenetic, ICSI, androgenetic, ROSI, fES-NT, and pES-NT embryos at the two-cell and four-cell stages. Embryos were measured at 24 and $48 \mathrm{~h}$ after sperm injection or activation for the two-cell and four-cell stages respectively. Nuclei for each developmental stage were classified into three groups. The fluorescence intensity observed for each sample was expressed relative to this value as $<20 \%$ indicating repressive transcription; $20-60 \%$ indicating low transcription; or $>60 \%$ indicating high transcription. Data were analyzed with respect to the proportion of transcriptionally active nuclei. 


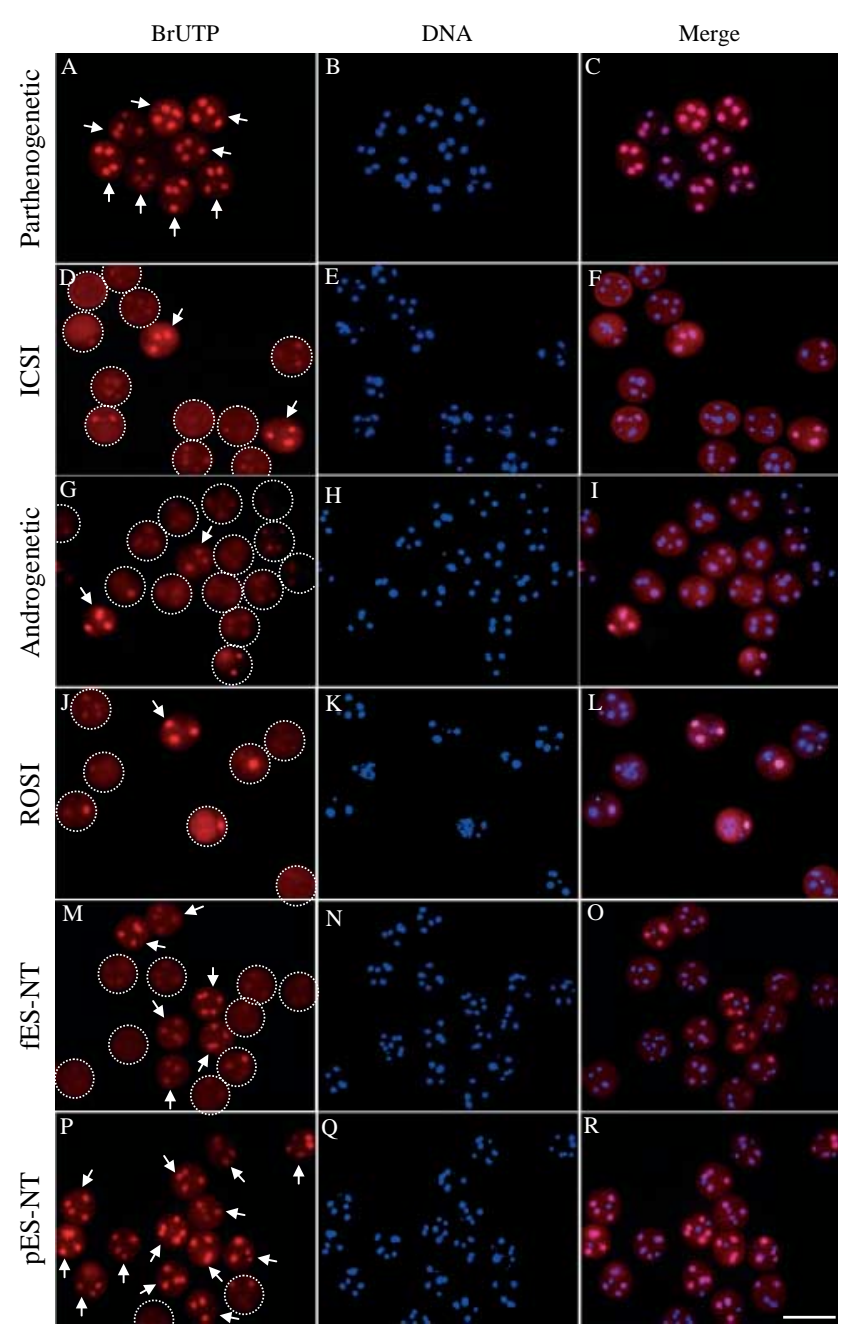

Figure 3 Expression levels of nascent RNA at the four-cell stage in (A-C) parthenogenetic, (D-F) ICSI, (G-I) androgenetic, (J-L) ROSI, (M-O) fES-NT, and (P-R) pES-NT embryos. Embryos selected at $48 \mathrm{~h}$ were labeled with BrUTP and collected for the detection of BrU-labeled transcripts using immunofluorescence microscopy. Nascent RNA is shown in red. The DNA was counterstained with DAPI (blue). The arrows indicate high levels of nascent RNA production in embryos and circles indicate low levels. Bar $=100 \mu \mathrm{m}$.

we examined whether different stages of sperm development could affect nascent RNA production, as differences in chromatin structure between spermatids and mature sperm might affect the later epigenetic status of the paternally inherited genome in the embryo (Kimmins \& Sassone-Corsi 2005). Therefore, matured oocytes were subjected to round spermatid injection (ROSI), cultured to the two-cell and four-cell stages, and the levels of nascent RNA produced were examined. Surprisingly, a high level of newly synthesized RNA was detected in only $23 \%$ of nuclei in the two-cell ROSIgenerated embryos, significantly less than in ICSIgenerated embryos (Figs 1J and 2). This indicated that the round spermatid has a stronger inhibiting effect than mature spermatozoa on nascent RNA production in twocell embryos (Fig. 2). Mouse embryos at this stage have clearly begun a transition from maternal to zygotic control of development, as shown by an increase in the abundance of specific RNAs at the two-cell stage (Conover et al. 1991). Therefore, the production of nascent RNA for protein synthesis in early zygotic gene activation is important for the successful development of embryos. That the ROSI-generated embryos exhibited a low level of transcriptional activity at this stage compared with those generated from mature spermatozoa by ICSI could be related to epigenetic abnormalities in ROSI-generated embryos, as reported previously (Kishigami et al. 2006). However, ROSIgenerated embryos also showed a low level of nascent RNA at the four-cell stage, similar to ICSI-generated embryos (Figs 2 and 3J). Therefore, male-derived factors must have been involved in the suppression of nascent RNA production during the two-cell to four-cell transition.

\section{Expression of nascent RNA in parthenogenetic, ICSI- generated, androgenetic, and ROSI-generated embryos after the four-cell transition}

We showed above that the nascent RNA production patterns are different during the zygotic gene activation stage for parthenogenetic, ICSI-generated, androgenetic, and ROSI-generated embryos. Next, we examined the level of nascent RNA production at later stages in these embryos.

First, we examined the differences in nascent RNA localization in the two-cell and four-cell ICSI-generated embryos. Using serial confocal microscopy sectioning, we found that four-cell embryos have accumulations of nascent RNA concentrated around the nucleoli (Fig. 4Ae). This localization differed from the two-cell embryos, which showed nascent RNA production uniformly throughout their nuclei (Fig. 4Ab). Thus, the two-cell to four-cell transition resulted in a repression of nascent RNA production in some regions of the nuclei (Fig. 4Ad-f). It was reported that in natural fertilization of mouse embryos, pericentric DNA dissociated from the periphery of the nucleolus and became colocalized into pro-chromocenters at the two-cell stage; however, pericentric DNA often did not colocalize with the whole pro-chromocenter, but only with parts of it at the four-cell stage (Martin et al. 2006). This change in chromatin structure during the two-cell to four-cell transition coincided with the repression of nascent RNA production in our results (Fig. 4Aa and d). However, in parthenogenetic embryos lacking male chromatin this pattern of nascent RNA was not found until the late four-cell stage.

At the late four-cell stage, nascent RNA production showed a slight increase in ICSI-generated embryos. However, in parthenogenetic embryos the levels 

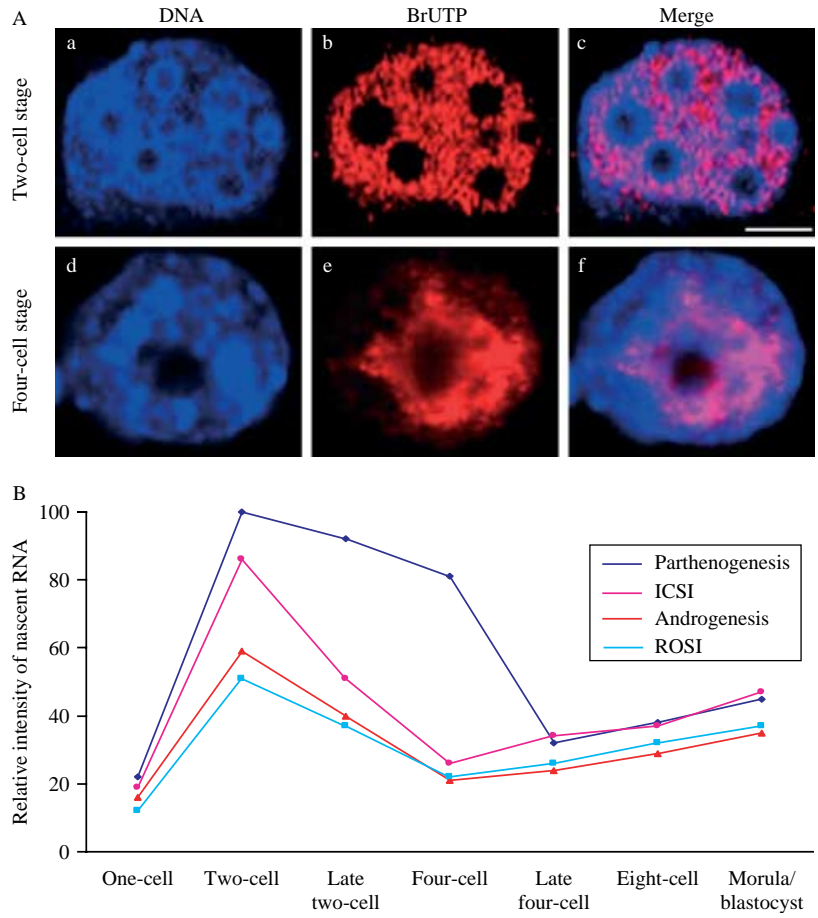

Figure 4 (A) Localization of nascent RNA in two-cell and four-cell nuclei of ICSI-generated embryos. Embryos selected at 24 and $48 \mathrm{~h}$ after ICSI were labeled with BrUTP and collected for the detection of BrU-labeled transcripts by serial optical sectioning using confocal microscopy. Nascent RNA is shown in red. The DNA was counterstained with DAPI (blue). (a-c) Distribution of nascent RNA was uniform throughout the nuclei at the two-cell stage. $(d-f)$ There was an accumulation of nascent RNA production associated with the nucleoli at the four-cell stage. Bar $=10 \mu \mathrm{m}$. (B) Nascent RNA production patterns in parthenogenetic, ICSI, androgenetic, and ROSI embryos. They were measured at 14, 24, 32, 48, 54, 62, and $84 \mathrm{~h}$ after sperm injection or activation for the one-cell, two-cell, late two-cell, four-cell, late four-cell, eight-cell, and morula/blastocyst stages respectively. Data were analyzed with respect to the relative intensity of nascent RNA.

decreased quickly in most of the embryos and reached levels similar to those seen in ICSI-generated embryos (Fig. 4B). Although transcriptional repression occurred in parthenogenetic embryos at this stage, it occurred later than in ICSI-generated embryos. In androgenetic and ROSI-generated embryos, nascent RNA production increased slightly in the late four-cell stage, reaching levels similar to control ICSI-generated embryos (Fig. 4B).

There were also accumulations of nascent RNA associated with the nucleoli in the nuclei of most embryos at the eight-cell stage, as in the four-cell stage (Fig. 5). Finally, nascent RNA levels increased slightly at the morula/blastocyst stage in each type of embryo (Figs 4B and 5). Thus, there was no significant difference in nascent RNA production patterns after the late four-cell stage between parthenogenetic and ICSIgenerated embryos.

\section{Differences between male and female chromatin remodeling, DNA synthesis, nascent RNA production, and histone modifications}

Our results have clearly shown that male-derived factor(s) play a key role in the regulation of transcriptional repression in the two-cell to four-cell transition; however, the paternal chromatin contribution to this process is unknown. We examined chromatin remodeling in male and female pronuclei along with DNA replication, nascent RNA production, acetylation of histone $\mathrm{H} 4$ at lysine 12 (Ac-H4-K12), and methylation of histone $\mathrm{H} 3$ at lysine $9(\mathrm{Me}-\mathrm{H} 3-\mathrm{K} 9)$ in early ICSIgenerated embryos. The latter measure allowed us to

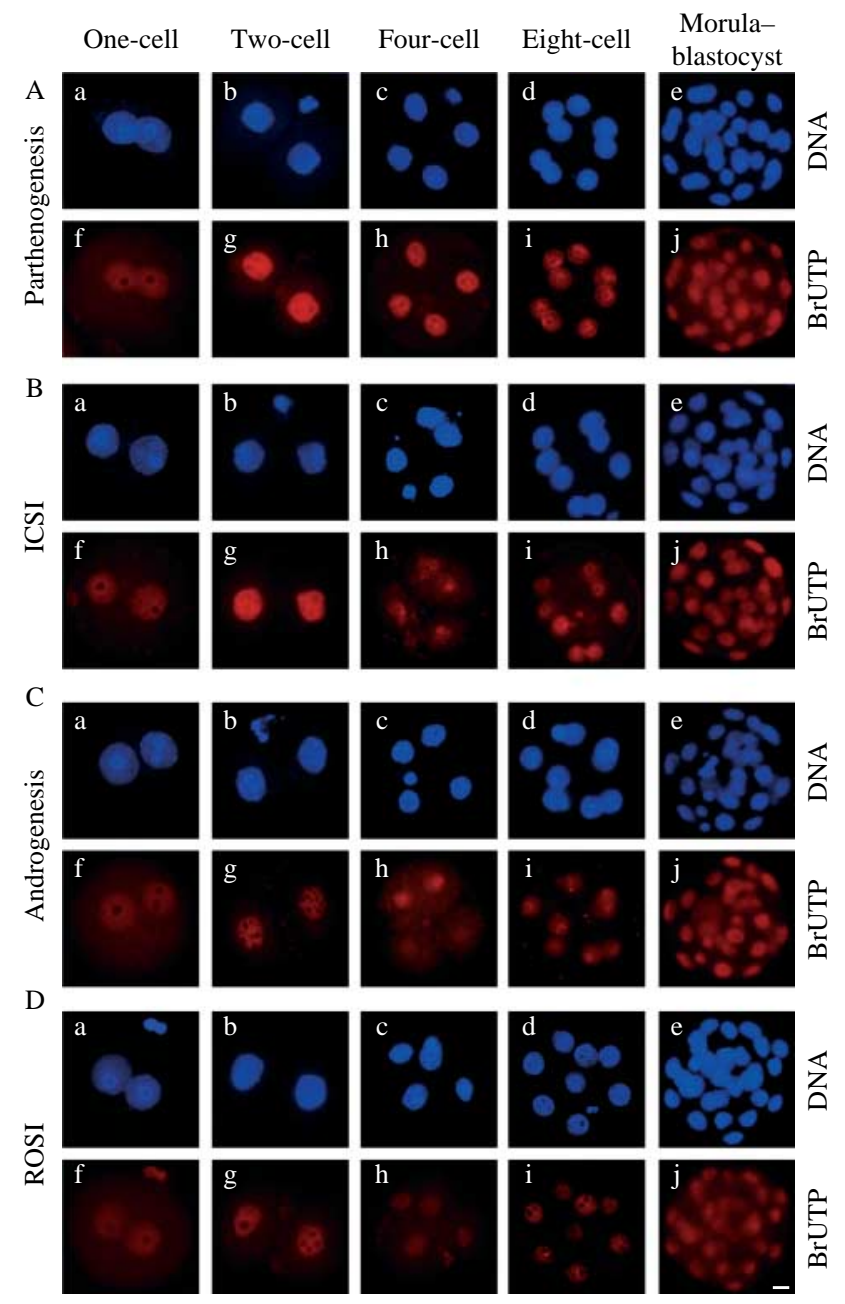

Figure 5 Expression levels of nascent RNA production in (A) parthenogenetic, (B) ICSI, (C) androgenetic, and (D) ROSI embryos. Embryos were measured at 14, 24, 48, 62, and $84 \mathrm{~h}$ after sperm injection or activation for the $(\mathrm{a}, \mathrm{f})$ one-cell, $(\mathrm{b}, \mathrm{g})$ two-cell, $(\mathrm{c}, \mathrm{h})$ four-cell, $(d, i)$ eight-cell, and $(e, j)$ morula/blastocyst stages respectively. Embryos were labeled with BrUTP and collected for the detection of BrU-labeled transcripts using immunofluorescence microscopy. Nascent RNA is shown in red. The DNA was counterstained with DAPI (blue). Bar $=10 \mu \mathrm{m}$. 
distinguish between the male (unlabeled) and female (labeled) pronuclei.

Initially, DNA replication occurred uniformly in the intranuclear region, and then only in the peripheral regions of the nucleus and nucleolus in both the male and female pronuclei (Fig. 6A). However, DNA synthesis in the peripheral regions differed in the timing and locality of completion. Thus, it terminated in the perinuclear region in the female pronuclei but in the perinucleolar region in the male pronuclei (Fig. 6Ad). By contrast, synthesis remained localized in the perinucleolar region in the female pronuclei and in the perinuclear region in the male pronuclei. DNA synthesis was also initiated earlier in the male than in the female pronuclei. The typical pattern of DNA replication in the male pronucleus started at $4 \mathrm{~h}$ after ICSI (data not shown), reached a high level at $5 \mathrm{~h}$, and finished at $9 \mathrm{~h}$ (Fig. 6A). However, the female pronuclei started DNA replication later than the male pronuclei and took longer to complete it (Fig. 6A).

Soon after ICSI, the paternal pronucleus expanded quickly and showed a high level of acetylation of $\mathrm{H} 4-\mathrm{K} 12$, whereas the maternal pronucleus was smaller, with weak staining for acetylation (Fig. 6Bb; 100\%; $n=60$ ). Nascent RNA production was detected at $10 \mathrm{~h}$ after ICSI in some embryos (data not shown). At $12 \mathrm{~h}$, the level of nascent RNA in the paternal pronucleus was also higher than in the maternal pronucleus (Fig. 6BC). There was a positive association between the level of nascent RNA production and histone acetylation in the one-cell ICSI-generated zygotes. However, there were no differences in transcriptional activity or histone acetylation among the parthenogenetic and androgenetic embryos at one-cell stage (Supplementary Figure 1A, see section on supplementary data given at the end of this article).

Next, we examined histone $\mathrm{H} 3$ methylation in the early ICSI-generated embryos. At the one-cell stage, embryos showed a high level of histone H3-K9 methylation in the maternal chromatin, whereas the paternal chromatin showed hypomethylation (Fig. 5Cb). This is consistent with the report of Arney et al. (2002) that global histone demethylation occurs in the paternal pronucleus. Interestingly, we found that at the two-cell stage, male chromatin (not labeled with Me-H3-K9) and female chromatin (labeled with Me-H3-K9) contributed separately to show different patterns of histone methylation (Fig. 6Ce). However, by the four-cell stage the male and female chromatin had mixed, leading to histone H3-K9 methylation in all of the chromatin (Fig. 6Ch). This combination of the male and female chromatin might induce a rapid decrease in nascent
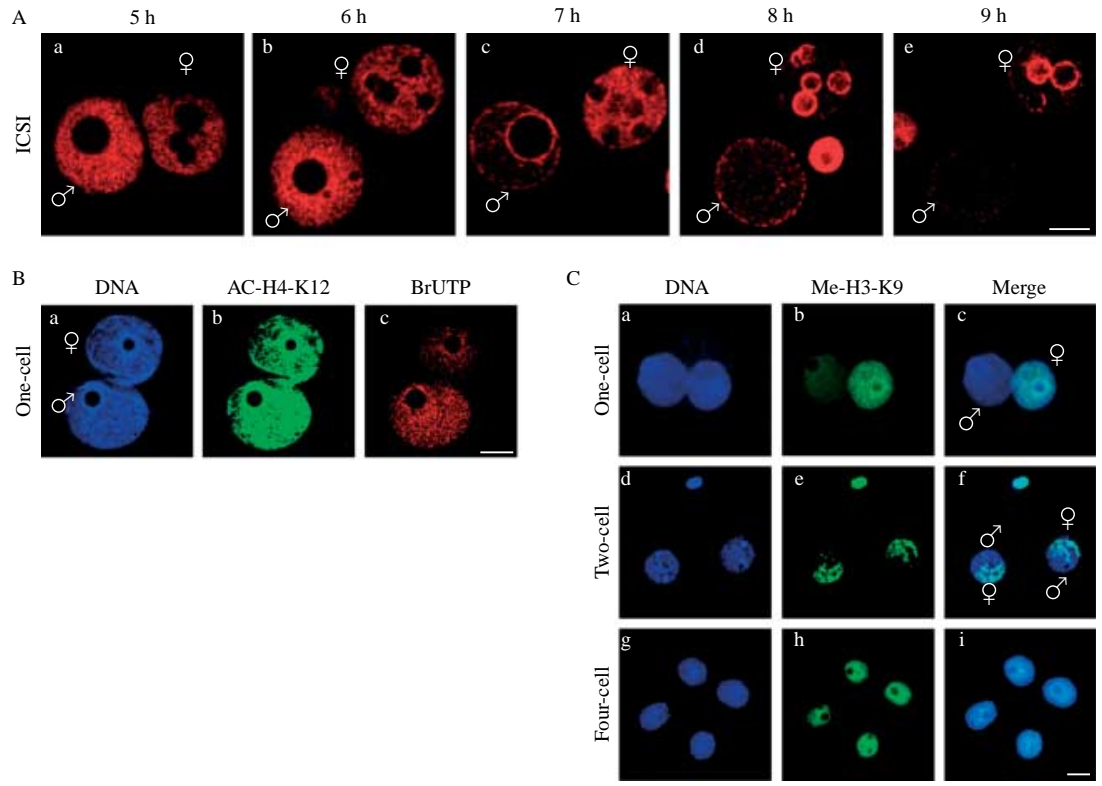

Figure 6 (A) Distribution of the sites of DNA replication in one-cell ICSI embryos (a-e). Embryos were incubated with BrdU for 30 min at 5, 6, 7, 8, and $9 \mathrm{~h}$, then collected for the detection of BrdU labeling using immunofluorescence microscopy. (B) One-cell embryos at $12 \mathrm{~h}$ after ICSI were labeled with BrUTP and collected for the detection of BrU-labeled transcripts and acetylation of H3-K12 using immunofluorescence microscopy. $(\mathrm{a}, \mathrm{b})$ The paternal pronucleus expanded quickly and showed a high level of acetylation, whereas the maternal pronucleus was smaller with weak acetylation. $(\mathrm{a}, \mathrm{c})$ The level of nascent RNA production in the male pronucleus was also higher than in the female one. Histone $\mathrm{H} 4$ acetylation at $\mathrm{K} 12$ is shown in green and nascent RNA is shown in red. The DNA was stained with DAPI (blue). (C) Expression of methylation level of histone $\mathrm{H} 3$ at $\mathrm{K} 9$ in paternal and maternal chromatin. (a-c) One-cell embryos showed a high level of histone H3-K9 methylation in the maternal chromatin, whereas the paternal chromatin showed hypomethylation. (d-f) Two-cell embryos showed that the male chromatin (not labeled with Me-H3-K9) and female chromatin (labeled with Me-H3-K9) contributed separately to show different patterns of histone methylation. (g-i) Four-cell embryos showing that both the male and female chromatin exhibited histone $\mathrm{H} 3-\mathrm{K} 9$ methylation at this stage of development. Bar $=10 \mu \mathrm{m}$. 
RNA production during the two-cell to four-cell transition in ICSI-generated embryos. The control groups showed a high level of histone $\mathrm{H} 3-\mathrm{K} 9$ methylation in the parthenogenetic embryos, whereas the androgenetic embryos showed hypomethylation at the one-cell and two-cell stages (Supplementary Figure 1B). These confirm that half of the staining of nuclei at the twocell stage arose from the female pronucleus. However, the asymmetry of histone $\mathrm{H} 3-\mathrm{K} 9$ methylation was lost at the four-cell stage. Uniform methylation fluorescence was observed in the nuclei of the four-cell stage in parthenogenetic and androgenetic embryos (Supplementary Figure 1B).

\section{Discussion}

Using BrUTP-labeled RNA, we demonstrated that nascent RNA production was repressed at the two-cell to four-cell transition in fertilized mouse embryos and that this repression was delayed in parthenogenetic embryos lacking a paternal genome. We confirmed that transcriptional repression during this stage was contributed by the spermatozoon or by male-derived chromatin. In addition, we also showed that mature spermatozoa could regulate transcriptional activity successfully during preimplantation development. Importantly, nascent RNA synthesis became repressed at the two-cell to four-cell transition in androgenetic as well as ICSI- and ROSI-generated embryos. We suggest that paternal chromatin causes transcriptional repression at this stage of development. Surprisingly, these male factors in fES-NT cloned embryos induced repression of nascent RNA transcription, whereas cloned embryos reconstructed with $\mathrm{pES}$ cell nuclei did not show repression during this phase. This suggests that one essential role of paternal chromatin in the mouse preimplantation embryo is the regulation of transcriptional activity during early embryogenesis.

Following sperm entry into the oocyte, immediate transcriptional activity was associated with the paternal but not with the maternal chromatin. This induced transcriptional activity was also higher in the male than in the female pronucleus. This is consistent with a report that transcriptional activity is higher in the male than in the female pronucleus in one-cell mouse embryos (Aoki et al. 1997). Transmission of histone $\mathrm{H} 4$ acetylation from the sperm might constitute the first phase (van der Heijden et al. 2006) and sperm-derived nucleosomal chromatin appears to contribute to paternal zygotic chromatin, potentially serving as a template for replication (van der Heijden et al. 2008). This increased histone acetylation was probably associated with more effective commencement of DNA replication in the male pronucleus, while the lower level of acetylation in the female pronucleus could be associated with a delay in completing DNA replication. The level of histone acetylation could have a direct effect on the timing of DNA replication in the embryo, as lower acetylation levels delay and high levels stimulate replication (Kemp et al. 2005). However, though histone acetylation is believed to lead to the initiation of gene transcription, it has also been suggested that histone H3-K9 methylation levels are correlated with gene silencing (Schotta et al. 2004, Papp \& Muller 2006). Histone H3-K9 trimethylation is only attributed to the maternal genome and is completely absent from the paternal genome in the zygote. These differences between male and female genomes seem to arise from the more efficient initial competition by paternal chromatin for the maternally derived RNA in the cytoplasm and from the maintenance of different levels of transcriptional activity in the female and male pronuclei. However, in parthenogenetic embryos, the maternal chromatin showed the same initial level of transcriptional activity in both female pronuclei. In addition, the injection of round spermatids produced the same level of transcriptional activity in both female and male pronuclei (Fig. 5Df). This indicates that the mechanism for repressing maternal transcriptional activity must involve the presence of a fertilizing spermatozoon. However, in androgenetic embryos lacking any maternal chromatin, the sperm genome did not express high transcriptional activity in either of the male pronuclei, as seen for the paternal pronucleus in ICSI-generated embryos. As the total amounts of BrUTP incorporation in ICSI- and ROSI-generated, parthenogenetic, and androgenetic zygotes were similar at the one-cell stage (Figs $4 \mathrm{~B}$ and 5), the transcriptional capacity might be inherently rate limiting during this stage. If not, an increase in the total amount of BrUTP should have been observed in androgenetic embryos. This limited ability for DNA transcription could be explained by the limited capacity of the one-cell embryo to form mature nuclei - in which the chromatin is completely remodeled - because of the limited pool of maternally derived histones (Clarke \& Masui 1986, 1987). In addition, we found that mature spermatozoa could regulate transcriptional activity successfully at an early embryonic stage because embryos produced by ROSI exhibited a low level of transcriptional activity at the two-cell stage compared with those generated with ICSI using mature spermatozoa. Similarly, we showed that cloned embryos produced by SCNT also exhibited a low level of transcriptional activity during the two-cell stage and that the enhanced nascent RNA production at this stage induced by chemical treatment increased the rate of full-term development of cloned mice (Van Thuan et al. 2009). This suggests that the poor full-term development of ROSI-generated embryos might result from aberrant transcriptional activity during this stage. This study opens the way to improve the full-term development of ROSI-generated embryos. The results also indicate that the male germ cell acquires the ability to maintain later transcriptional 
activity in the paternal zygotic genome during spermiogenesis.

It has been suggested that the formation of a transcriptionally repressive state at the two-cell stage is superimposed on activation of the embryonic genome in the preimplantation mouse embryo (Majumder et al. 1993, Aoki et al. 1997). Accordingly, in our experiments nascent RNA transcription decreased in late two-cell embryos and was repressed in four-cell embryos. This is consistent with the suggestion that gene repression occurs between the two-cell and four-cell stages in the mouse embryo (Ma et al. 2001). From our data and previous reports, it appears that paternal genome must be involved in activation of the embryonic genome.

Interestingly, cloned embryos reconstructed with pES-NT cells showed nascent RNA production patterns that were similar to parthenogenetic embryos. It is well known that parthenogenetic embryos lack the potential to develop to full term, as the paternal genome is missing; they invariably die in mid-gestation from imprinted gene defects and placental hypoplasia. Based on chimera experiments, trophoblastic proliferation is supposed to be inhibited in the absence of a male genome. It is suggested that the contribution rate of the pES cells to chimeric offspring was very low and offspring often died soon after birth (Allen et al. 1994, Hikichi et al. 2007, Chen et al. 2009). In addition, although trophoblastic stem cells - the precursors of placental tissues - can be established from fertilized and androgenetic embryos, they have never been derived from parthenogenetic embryos (Ogawa et al. 2009). Taken together, these results suggest that the paternal chromatin is important not only in genomic silencing during zygotic gene activation but also for the full-term development of embryos.

Our data revealed that the paternal chromatin is important in the regulation of transcriptional activity during mouse preimplantation embryogenesis, and the mechanism for repressing maternal transcriptional activity must involve the presence of a fertilizing spermatozoon. Much remains to be learned about the mechanisms involved in the reprograming of sperm chromatin, which modulate transcriptional activity. Therefore, studies on the role of transcription factors in the fertilizing spermatozoon and the paternal pronucleus are imperative if we are to understand these epigenetic processes in embryogenesis.

\section{Materials and Methods \\ Animals}

B6D2F1 (C57BL/6J $\times \mathrm{DBA} / 2)$ strain mice were obtained at 8-10 weeks of age from SLC (Hamamatsu, Japan). All animal experiments conformed to the Guide for the Care and Use of Laboratory Animals and were approved by the Institutional
Committee of Laboratory Animal Experimentation of the Center for Developmental Biology, RIKEN-Kobe, Japan.

\section{Oocyte collection}

Female B6D2F1 mice (8-10-weeks old) were superovulated by the administration of $5 \mathrm{IU}$ equine chorionic gonadotropin followed $48 \mathrm{~h}$ later by $5 \mathrm{IU}$ human chorionic gonadotropin (hCG). Oocytes were collected from the oviducts about $16 \mathrm{~h}$ after the hCG injection. After collection, cumulus cells were dispersed with $0.1 \%$ hyaluronidase (Sigma-Aldrich) in droplets of HEPES-buffered CZB medium (HEPES-CZB; Chatot et al. 1989). After several minutes, the oocytes were transferred to fresh droplets of HEPES-CZB and were denuded of almost all cumulus cells by gentle pipetting. Denuded oocytes with a homogeneous ooplasm were selected and resuspended in new droplets of potassium simplex optimized medium (KSOM) with amino acids; Specialty Media, Phillipsburg, NJ, USA) containing 1\% BSA (Sigma-Aldrich), covered earlier with equilibrated paraffin oil (Nacalai Tesque, Kyoto, Japan). The oocytes were then cultured at $37^{\circ} \mathrm{C}$ in a $5 \% \mathrm{CO}_{2}$ atmosphere until use.

\section{Microinjection with spermatozoa and round spermatids}

Fertilized embryos were produced using ICSI or ROSI. ICSI was performed as described by Kimura \& Yanagimachi (1995) except that our experiments were performed at room temperature. Briefly, after washing, the sperm heads were separated from the tail by subjecting the head-tail junction to a few pulses using a piezo-activated micromanipulator system (Prime Tech, Aburaki, Tokyo, Japan). Only the sperm head was injected into each oocyte. After 10-20 min of recovery, the ICSI-generated embryos were cultured in KSOM at $37{ }^{\circ} \mathrm{C}$ in humidified air with $5 \% \mathrm{CO}_{2}$ for preimplantation development. ROSI was carried out as described by Kishigami et al. (2006). Briefly, oocytes were activated by incubation in $\mathrm{Ca}^{2+}$-free CZB medium containing $5 \mathrm{mM} \mathrm{SrCl}_{2}$ for $20 \mathrm{~min}$ and transferred into the $\mathrm{KSOM}$ medium at $37{ }^{\circ} \mathrm{C}$ in a $5 \%$ $\mathrm{CO}_{2}$ incubator. Round spermatids, characterized by their small size (about $10 \mu \mathrm{m}$ ) and a centrally located distinct nucleolus, were injected individually into 12-15 activated oocytes between 50 and 80 min after oocyte activation. After 10-20 min of recovery, the ROSI-generated embryos were cultured in the KSOM medium as described above.

\section{Androgenesis and parthenogenesis}

Oocyte metaphase stage II (MII) chromosomes were removed by aspiration as described by Wakayama et al. (1998). Each enucleated oocyte was injected with two sperm heads as described above. These androgenetic embryos were cultured in KSOM for preimplantation development as above. Parthenogenetically activated oocytes were prepared by the exposure of MIl oocytes for $6 \mathrm{~h}$ to $5 \mathrm{mM} \mathrm{SrCl}_{2}$ in $\mathrm{Ca}^{2+}$-free CZB medium with $5 \mu \mathrm{g} / \mathrm{ml}$ of cytochalasin B (Kishigami \& Wakayama 2007). Following activation and cytochalasin B treatment, they were washed and cultured in the KSOM medium as above. 


\section{Establishment of fES and pES cells}

Fertilized or parthenogenetic embryos at the morula or blastocyst stages were used to establish ES cell lines as described (Wakayama et al. 2001, Ogawa et al. 2004, Wakayama 2006) with a slight modification in that $20 \%$ knockout serum replacement (Invitrogen) and $0.1 \mathrm{mg} / \mathrm{ml}$ ACTH (American Peptide Company, Sunnyvale, CA, USA) were added to the multiES cell medium instead of FCS (Ogawa et al. 2004). Briefly, morulae/blastocysts were treated with acid Tyrode's solution to remove the zona pellucida and placed in 96-multiwell dishes coated with mouse embryonic fibroblasts (from ICR mice) for at least 10 days. Proliferating outgrowths were dissociated using trypsin digestion and replated on the fibroblasts until stable cell lines grew out. The culture medium was replaced with the cryopreservation medium Bambanker (Nippon Genetics, Tokyo, Japan) and the cell lines were stored in a $-80{ }^{\circ} \mathrm{C}$ freezer soon after being established.

\section{Nuclear transfer}

Cloning by SCNT and ESNT was performed as described by Wakayama et al. (1998, 1999). Briefly, fES or pES cells were thawed and cultured for 2 days. Individual fES or pES cells were collected from flasks by trypsinization for $5 \mathrm{~min}$. To remove the trypsin, cells were washed at least twice in $5 \mathrm{ml}$ of PBS (Sigma-Aldrich) and finally $2 \mu \mathrm{l}$ of condensed cell suspension was transferred to HEPES-CZB containing $12 \%$ $(\mathrm{w} / \mathrm{v})$ polyvinylpyrrolidone $\left(M_{\mathrm{r}} 360\right.$ 000; Sigma-Aldrich). Oocytes were collected from BDF1 females and MII spindles were removed as described by Wakayama et al. (1998). For nuclear injection, enucleated oocytes were transferred to the micromanipulation chamber and then each fES or pES cell was drawn in and out of the injection pipette until the cell membrane was broken. In some cases, a few piezo pulses were applied to break the plasma membrane. Several nuclei were drawn into a pipette individually and injected individually into separate enucleated oocytes at room temperature. After NT, the reconstructed oocytes were activated by exposure to $10 \mathrm{mM} \mathrm{SrCl}_{2}$ in $\mathrm{Ca}^{2+}$-free $\mathrm{CZB}$ medium in the presence of $5 \mu \mathrm{g} / \mathrm{ml}$ cytochalasin B (Kishigami \& Wakayama 2007). Following activation and cytochalasin B treatment, they were washed and cultured in the KSOM medium as above. Control cloned embryos were generated using BDF1 cumulus cells for SCNT as above.

\section{Detection of DNA synthesis}

DNA synthesis was measured from the incorporation of 5-bromo-deoxyuridine (BrdU; Sigma-Aldrich). Embryos were incubated with $10 \mu \mathrm{M}$ BrdU for $30 \mathrm{~min}$ under $5 \% \mathrm{CO}_{2}$ in humidified air at $37^{\circ} \mathrm{C}$. Embryos were incubated from $15 \mathrm{~min}$ before to $15 \mathrm{~min}$ after each time point following pronuclear formation. The embryos were then fixed in $4 \%$ paraformaldehyde and DNA synthesis incorporating BrdU was visualized by indirect immunofluorescence microscopy.

\section{Incorporation of BrUTP into embryos and detection of new transcripts}

Each group of embryos $(<20)$ was washed twice in electrical permeabilization medium (EP: $0.25 \mathrm{M}$ D-glucose, $100 \mu \mathrm{M}$ $\mathrm{CaCl}_{2} \cdot 2 \mathrm{H}_{2} \mathrm{O}, 100 \mu \mathrm{M} \mathrm{MgSO}_{4}$, and $0.1 \%$ polyvinylpyrrolidone) and finally in one change of transcription buffer $(\mathrm{EP}+10 \mathrm{mM}$ BrUTP; Sigma-Aldrich). They were then transferred into a chamber between electrodes overlaid with a $20 \mu \mathrm{l}$ droplet of transcription buffer. Two $80 \mu$ s electric pulses at $250 \mathrm{~V} / \mathrm{cm}$ of direct current were triggered using an Electro Cell Fusion apparatus (Bex LF101L, Tokyo, Japan) with a 2 min interval between pulses. Two minutes after permeabilization, embryos were cultured in the CZB medium for $1 \mathrm{~h}$ further under the same conditions as above. The embryos were then fixed in $4 \%$ paraformaldehyde and new transcripts incorporating BrUTP were visualized by indirect immunofluorescence. In a negative control group, $5 \mu \mathrm{g} / \mathrm{ml}$ of $\alpha$-amanitin (Sigma-Aldrich), an inhibitor of RNA polymerases II and III, was added to the EP medium, transcription buffer, and KSOM medium. Embryos were activated and cultured as described above.

\section{Immunofluorescence microscopy}

Paraformaldehyde-fixed embryos were treated as described by Bui et al. (2004). The primary antibodies used were rabbit polyclonal anti-histone $\mathrm{H} 4$ at lysine 12 (Ac-H4-K12; Upstate Cell Signaling Solutions, Charlottesville, VA, USA), rabbit anti-trimethyl-histone $\mathrm{H} 3$ at lysine 9 (Me-H3-K9; Abcam, Cambridge, UK), and mouse monoclonal antiBrdU (Roche Diagnostics $\mathrm{GmbH}$ ). The secondary antibodies were Alexa-Fluor-568-labeled goat anti-mouse IgG or Alexa-Fluor-488-labeled chicken anti-rabbit IgG antibodies (Molecular Probes, Inc., Eugene, OR, USA). The DNA was stained with $2 \mu \mathrm{g} / \mathrm{ml}$ 4,6-diamidino-2-phenylindole (DAPI; Molecular Probes). For detecting DNA synthesis, before incubation with the first antibody fixed embryos were treated with $2 \mathrm{M} \mathrm{HCl}$ at room temperature for $1 \mathrm{~h}$, neutralized for $20 \mathrm{~min}$ in $100 \mathrm{mM}$ Tris/ $\mathrm{HCl}$ ( $\mathrm{pH} \mathrm{8.0),} \mathrm{and}$ washed extensively with PBS containing 1\% BSA. Subsequent steps followed the standard protocol for immunofluorescence as described above.

\section{Quantitation of fluorescence intensity in nuclei}

Embryos were observed using an Olympus Fluoview FV1000 confocal scanning laser microscope (Olympus, Tokyo, Japan). Fluorescence intensities of nuclei were measured by manually outlining all nuclei in the display as described by Bui et al. (2008). Briefly, nuclei were selected randomly except for the premetaphase or metaphase stages as shown in Supplementary Figure $2 \mathrm{Ab}$ and $\mathrm{Ac}$ respectively (see section on supplementary data given at the end of this article). The total intensity in each nucleus was measured from five different regions and the background value for the cytoplasm was subtracted. This calculated intensity was multiplied by the nuclear volume to estimate the total amount of fluorescence for the nucleus. One-cell embryos were measured at 10, 12, and $14 \mathrm{~h}$ after sperm injection or activation. For two-cell, late two-cell, 
four-cell, late four-cell and eight-cell, and morula/blastocyst stages, embryos were measured at 24, 32, 48, 54, 62, and $84 \mathrm{~h}$ after sperm injection or activation respectively.

\section{Statistical analysis}

Student's t-test was used to estimate the significance of any differences between the experimental groups in the immunofluorescence studies. Each experiment was repeated at least 3 times and about 100 immunostained oocytes were examined in each group. The data were subjected to arcsine transformation for each replication to normalize them. The transformed values were analyzed using one-way ANOVA and $P<0.05$ was considered statistically significant.

\section{Supplementary data}

This is linked to the online version of the paper at http://dx.doi. org/10.1530/REP-10-0109.

\section{Declaration of interest}

The authors declare that there is no conflict of interest that could be perceived as prejudicing the impartiality of the research reported.

\section{Funding}

Support for this research was provided by a Scientific Research in Priority Areas (15080211) grant to T Wakayama for the realization of regenerative medicine in Japan (the research field for the technical development of stem cell manipulation); by the BioGreen21 program of the RDA (grants PJ007189 and PJ007065) and by the 2009 KU Brain Pool Program of Konkuk University, Republic of Korea to H-T Bui.

\section{Acknowledgements}

We are grateful to the Laboratory for Animal Resources and Genetic Engineering of RIKEN-KOBE, Center for Developmental Biology for the housing of mice.

\section{References}

Allen ND, Barton SC, Hilton K, Norris ML \& Surani MA 1994 A functional analysis of imprinting in parthenogenetic embryonic stem cells. Development 120 1473-1482.

Aoki F, Worrad DM \& Schultz RM 1997 Regulation of transcriptional activity during the first and second cell cycles in the preimplantation mouse embryo. Developmental Biology 181 296-307. (doi:10.1006/ dbio.1996.8466)

Arney KL, Bao S, Bannister AJ, Kouzarides T \& Surani MA 2002 Histone methylation defines epigenetic asymmetry in the mouse zygote. International Journal of Developmental Biology 46 317-320.

Barton SC, Arney KL, Shi W, Niveleau A, Fundele R, Surani MA \& Haaf T 2001 Genome-wide methylation patterns in normal and uniparental early mouse embryos. Human Molecular Genetics 10 2983-2987. (doi:10.1093/hmg/10.26.2983)
Bui HT, Yamaoka E \& Miyano T 2004 Involvement of histone H3 (Ser10) phosphorylation in chromosome condensation without Cdc2 kinase and mitogen-activated protein kinase activation in pig oocytes. Biology of Reproduction 70 1843-1851. (doi:10.1095/biolreprod.103.026070)

Bui HT, Wakayama S, Kishigami S, Kim JH, Van Thuan N \& Wakayama T 2008 The cytoplasm of mouse germinal vesicle stage oocytes can enhance somatic cell nuclear reprogramming. Development 135 3935-3945. (doi:10.1242/dev.023747)

Chatot CL, Ziomek CA, Bavister BD, Lewis JL \& Torres I 1989 An improved culture medium supports development of random-bred 1-cell mouse embryos in vitro. Journal of Reproduction and Fertility 86 679-688. (doi:10.1530/jrf.0.0860679)

Chen Z, Liu Z, Huang J, Amano T, Li C, Cao S, Wu C, Liu B, Zhou L, Carter MG et al. 2009 Birth of parthenote mice directly from parthenogenetic embryonic stem cells. Stem Cells 27 2136-2145. (doi:10.1002/stem.158)

Clarke HJ \& Masui Y 1986 Transformation of sperm nuclei to metaphase chromosomes in the cytoplasm of maturing oocytes of the mouse. Journal of Cell Biology 102 1039-1046. (doi:10.1083/jcb.102.3.1039)

Clarke HJ \& Masui Y 1987 Dose-dependent relationship between oocyte cytoplasmic volume and transformation of sperm nuclei to metaphase chromosomes. Journal of Cell Biology 104 831-840. (doi:10.1083/jcb. 104.4.831)

Conover JC, Temeles GL, Zimmermann JW, Burke B \& Schultz RM 1991 Stage-specific expression of a family of proteins that are major products of zygotic gene activation in the mouse embryo. Developmental Biology 144 392-404. (doi:10.1016/0012-1606(91)90431-2)

Davis W Jr, De Sousa PA \& Schultz RM 1996 Transient expression of translation initiation factor elF-4C during the 2-cell stage of the preimplantation mouse embryo: identification by mRNA differential display and the role of DNA replication in zygotic gene activation. Developmental Biology 174 190-201. (doi:10.1006/dbio.1996.0065)

van der Heijden GW, Derijck AA, Ramos L, Giele M, van der Vlag J \& de Boer P 2006 Transmission of modified nucleosomes from the mouse male germline to the zygote and subsequent remodeling of paternal chromatin. Developmental Biology 298 458-469. (doi:10. 1016/j.ydbio.2006.06.051)

van der Heijden GW, Ramos L, Baart EB, van den Berg IM, Derijck AA, van der Vlag J, Martini E \& de Boer P 2008 Sperm-derived histones contribute to zygotic chromatin in humans. BMC Developmental Biology 8 34. (doi:10.1186/1471-213X-8-34)

Henery CC, Miranda M, Wiekowski M, Wilmut I \& DePamphilis ML 1995 Repression of gene expression at the beginning of mouse development. Developmental Biology 169 448-460. (doi:10.1006/dbio.1995.1160)

Hikichi T, Wakayama S, Mizutani E, Takashima Y, Kishigami S, Van Thuan N, Ohta H, Thuy Bui H, Nishikawa $S$ \& Wakayama $T$ 2007 Differentiation potential of parthenogenetic embryonic stem cells is improved by nuclear transfer. Stem Cells 25 46-53. (doi:10.1634/ stemcells.2006-0439)

Kageyama S, Nagata M \& Aoki F 2004 Isolation of nascent messenger RNA from mouse preimplantation embryos. Biology of Reproduction $\mathbf{7 1}$ 1948-1955. (doi:10.1095/biolreprod.104.031906)

Kemp MG, Ghosh M, Liu G \& Leffak M 2005 The histone deacetylase inhibitor trichostatin A alters the pattern of DNA replication origin activity in human cells. Nucleic Acids Research 33 325-336. (doi:10. 1093/nar/gki177)

Kimmins S \& Sassone-Corsi P 2005 Chromatin remodelling and epigenetic features of germ cells. Nature 434 583-589. (doi:10.1038/ nature03368)

Kimura Y \& Yanagimachi R 1995 Intracytoplasmic sperm injection in the mouse. Biology of Reproduction 52 709-720. (doi:10.1095/biolreprod52.4.709)

Kishigami S \& Wakayama T 2007 Efficient strontium-induced activation of mouse oocytes in standard culture media by chelating calcium. Journal of Reproduction and Development 53 1207-1215. (doi:10. 1262/jrd.19067)

Kishigami S, Van Thuan N, Hikichi T, Ohta H, Wakayama S, Mizutani E \& Wakayama T 2006 Epigenetic abnormalities of the mouse paternal zygotic genome associated with microinsemination of round spermatids. Developmental Biology 289 195-205. (doi:10.1016/j.ydbio.2005. 10.026) 
Lee DY, Hayes JJ, Pruss D \& Wolffe AP 1993 A positive role for histone acetylation in transcription factor access to nucleosomal DNA. Cell 72 73-84. (doi:10.1016/0092-8674(93)90051-Q)

Ma J, Svoboda P, Schultz RM \& Stein P 2001 Regulation of zygotic gene activation in the preimplantation mouse embryo: global activation and repression of gene expression. Biology of Reproduction 64 1713-1721. (doi:10.1095/biolreprod64.6.1713)

Majumder S \& DePamphilis ML 1995 A unique role for enhancers is revealed during early mouse development. BioEssays 17 879-889. (doi:10.1002/bies.950171010)

Majumder S, Miranda M \& DePamphilis ML 1993 Analysis of gene expression in mouse preimplantation embryos demonstrates that the primary role of enhancers is to relieve repression of promoters. $E M B O$ Journal 12 1131-1140.

Martianov I, Brancorsini S, Gansmuller A, Parvinen M, Davidson I \& Sassone-Corsi P 2002 Distinct functions of TBP and TLF/TRF2 during spermatogenesis: requirement of TLF for heterochromatic chromocenter formation in haploid round spermatids. Development 129 945-955.

Martin C, Beaujean N, Brochard V, Audouard C, Zink D \& Debey P 2006 Genome restructuring in mouse embryos during reprogramming and early development. Developmental Biology 292 317-332. (doi:10.1016/ j.ydbio.2006.01.009)

Martinez-Salas E, Linney E, Hassell J \& DePamphilis ML 1989 The need for enhancers in gene expression first appears during mouse development with formation of the zygotic nucleus. Genes and Development 3 1493-1506. (doi:10.1101/gad.3.10.1493)

Mayer W, Niveleau A, Walter J, Fundele R \& Haaf T 2000 Demethylation of the zygotic paternal genome. Nature 403 501-502. (doi:10.1038/ 35000656)

Nothias JY, Majumder S, Kaneko KJ \& DePamphilis ML 1995 Regulation of gene expression at the beginning of mammalian development. Journal of Biological Chemistry 270 22077-22080. (doi:10.1074/jbc.270.38. 22077)

Ogawa K, Matsui H, Ohtsuka S \& Niwa H 2004 A novel mechanism for regulating clonal propagation of mouse ES cells. Genes to Cells 9 471-477. (doi:10.1111/j.1356-9597.2004.00736.x)

Ogawa H, Shindo N, Kumagai T, Usami Y, Shikanai M, Jonwn K, Fukuda A, Kawahara M, Sotomaru Y, Tanaka S et al. 2009 Developmental ability of trophoblast stem cells in uniparental mouse embryos. Placenta $\mathbf{3 0}$ 448-456. (doi:10.1016/j.placenta.2009.02.006)

Papp B \& Muller J 2006 Histone trimethylation and the maintenance of transcriptional ON and OFF states by trxG and PcG proteins. Genes and Development 20 2041-2054. (doi:10.1101/gad.388706)

Schotta G, Lachner M, Sarma K, Ebert A, Sengupta R, Reuter G, Reinberg D \& Jenuwein T 2004 A silencing pathway to induce H3-K9 and H4-K20 trimethylation at constitutive heterochromatin. Genes and Development 18 1251-1262. (doi:10.1101/gad.300704)
Stead E, White J, Faast R, Conn S, Goldstone S, Rathjen J, Dhingra U, Rathjen P, Walker D \& Dalton S 2002 Pluripotent cell division cycles are driven by ectopic Cdk2, cyclin A/E and E2F activities. Oncogene 21 8320-8333. (doi:10.1038/sj.onc.1206015)

Van Thuan N, Bui HT, Kim JH, Hikichi T, Wakayama S, Kishigami S, Mizutani E \& Wakayama T 2009 The histone deacetylase inhibitor scriptaid enhances nascent mRNA production and rescues full-term development in cloned inbred mice. Reproduction 138 309-317. (doi:10.1530/REP-08-0299)

Vettese-Dadey M, Grant PA, Hebbes TR, Crane-Robinson C, Allis CD \& Workman JL 1996 Acetylation of histone H4 plays a primary role in enhancing transcription factor binding to nucleosomal DNA in vitro. EMBO Journal 15 2508-2518.

Wakayama T 2006 Establishment of ES cell lines from adult somatic cells by nuclear transfer. In Cell Biology: A Laboratory Handbook, vol 1, pp 87-95. Ed. J Celis. San Diego, CA: Academic Press.

Wakayama T, Perry AC, Zuccotti M, Johnson KR \& Yanagimachi R 1998 Full-term development of mice from enucleated oocytes injected with cumulus cell nuclei. Nature 394 369-374. (doi:10.1038/28615)

Wakayama T, Rodriguez I, Perry AC, Yanagimachi R \& Mombaerts P 1999 Mice cloned from embryonic stem cells. PNAS 96 14984-14989. (doi:10.1073/pnas.96.26.14984)

Wakayama T, Tabar V, Rodriguez I, Perry AC, Studer L \& Mombaerts P 2001 Differentiation of embryonic stem cell lines generated from adult somatic cells by nuclear transfer. Science 292 740-743. (doi:10.1126/science. 1059399)

Wiekowski M, Miranda M \& DePamphilis ML 1991 Regulation of gene expression in preimplantation mouse embryos: effects of the zygotic clock and the first mitosis on promoter and enhancer activities. Developmental Biology 147 403-414. (doi:10.1016/0012-1606(91) 90298-H)

Wiekowski M, Miranda M, Nothias JY \& DePamphilis ML 1997 Changes in histone synthesis and modification at the beginning of mouse development correlate with the establishment of chromatin mediated repression of transcription. Journal of Cell Science 110 1147-1158.

Young LE \& Beaujean N 2004 DNA methylation in the preimplantation embryo: the differing stories of the mouse and sheep. Animal Reproduction Science 82-83 61-78. (doi:10.1016/j.anireprosci.2004. 05.020)

Received 25 February 2010

First decision 31 March 2010

Revised manuscript received 12 October 2010

Accepted 25 October 2010 